\title{
Spinodal Decomposition in Fluids
}

\author{
Martin Grant ${ }^{1}$ and K. R. Elder ${ }^{2}$ \\ ${ }^{1}$ Physics Department, McGill University, Rutherford Building, 3600 rue University, Montréal, Québec, Canada H3A 278 \\ ${ }^{2}$ Department of Physics, Oakland University, Rochester, Michigan 48309-4487
}

(Received 7 August 1998)

\begin{abstract}
If there exists an asymptotic scaling regime for spinodal decomposition in phase-separating fluids where domain sizes follow $L \sim t^{n}$, then, it is argued, the growth exponent $n$ is no larger than $1 / 2$. [S0031-9007(98)08124-1]
\end{abstract}

PACS numbers: 64.60.Ja, 64.60.My, 64.60.Cn, 81.30.Hd

During the late stages of spinodal decomposition, it has often been found that there is dynamical scale invariance $[1,2]$, where morphologies at different times can be related by a single characteristic length, the average domain size $L$. This length grows as a power law in time, i.e., $L \sim t^{n}$, where $n$ is the growth exponent. It is thought that a large number of systems can be grouped into a small number of universality classes, characterized by a few common features such as $n$, and the scaled form of the structure factor. The universality classes themselves are determined by, at least, the presence or absence of conservation laws for the ordering field. There is a strong similarity between these features and critical dynamics, where dynamical scaling occurs near a second-order transition [3]. Here, however, the scaling is very simple. For example, the growth exponents are usually independent of dimension, can be obtained from simple dimensional arguments, and bridge the nominal universality classes of critical dynamics. For scalar order parameters in the absence of hydrodynamic fields, it seems that the presence (giving $n=1 / 3$ ) or absence $(n=1 / 2)$ of a conservation law is the main feature determining the universality class. While the phenomenology of asymptotic scaling is well understood, much of it is based on the results of experiments or computer simulations, rather than fundamental theory.

The systems least well understood are fluids, although some scenarios for phase separation have been suggested [4-7] that have some experimental support [8-16]. Such systems potentially constitute a new universality class since velocity fields in fluids are due to Poisson-bracket relations, which are known to be relevant for determining universality classes in critical dynamics [3]. Recent advances in computer power have made these issues accessible to numerical simulations [17-29]. Indeed, simulations and dimensional arguments give various results for $n$, including $n=1$ and $n=2 / 3$. These are quite different from the result in the absence of velocity fields, $n=1 / 3$, and thus lead to the tantalizing possibility of a new universality class for spinodal decomposition. While some of these are effective exponents describing transient regimes, some are claimed to be asymptotic. It is important to note that only an asymptotic exponent can be used to describe a universality class.
The main issue involves the convective nonlinearity in the Navier-Stokes equation. The Reynolds number determines the relative importance of convective to viscous flow: $\operatorname{Re} \equiv \rho v \ell / \eta$, where $\eta$ is the shear viscosity, $v$ is the fluid velocity, $\ell$ is a characteristic length, and $\rho$ is the density. If an asymptotic scaling regime exists during phase separation in fluids, both $v$ and $\ell$ can be expressed in terms of the same time-dependent length $L(t)$. (The alternative is that there is no asymptotic scaling regime, so that $v$ and $\ell$ are not expressed in terms of the same length.) Then [22]

$$
\operatorname{Re} \sim t^{2 n-1} .
$$

For large times, however, the fluids phase separate. The Reynolds number cannot diverge, as this would imply turbulent remixing of the fluids when the critical Reynolds number $\mathrm{Re}_{\mathrm{cr}}$ is reached. Hence

$$
n \leq 1 / 2,
$$

which is the main result of this paper [23,30].

Nevertheless, values of $n$ have been reported for spinodal decomposition in phase separating fluids which do not satisfy this bound [31]. Many of these are simply effective exponents describing transient regimes [32] and are not addressed by the bound on the asymptotic exponent. This includes the theoretical result [4] $n_{\text {eff }}=1$ for the tubular necking down of an interconnected structure into droplets. However, one candidate that has been observed in many numerical simulations [17-23] and proposed as the asymptotic exponent [7], $n=2 / 3$, clearly violates the bound given above. This situation is reviewed below.

The exponent of $2 / 3$ is derived from dimensional analysis, which is not rigorous. The argument is as follows: Consider the Navier-Stokes equation, $\rho(\partial \vec{v} / \partial t+\vec{v}$. $\vec{\nabla} \vec{v})=-\vec{\nabla} p+\eta \nabla^{2} \vec{v}$, where $p$ is the pressure. In a liquid-vapor system, the excess pressure over a curved surface of radius $L$ is $\sigma / L$, where $\sigma$ is the surface tension. (Similar arguments can be made for a binary fluid [33].) The dimensional analysis assumes that scaling occurs and each additive term can be considered independently. For example, comparing the convective nonlinearity $(\rho \vec{v}$. $\vec{\nabla} \vec{v} \sim \rho L / t^{2}$ ) with the thermodynamic force due to pressure $\left(\nabla p \sim \sigma / L^{2}\right)$ gives $L \sim(\sigma / \rho)^{1 / 3} t^{2 / 3}$. Physically, 
the idea is that the convective flow of the fluid enhances the minimization of interface curvature. However, in accordance with the argument given above, at late enough times, the convective flow would be so strong as to remix the fluid. For example, one does not stir salad dressing to increase the size of oil droplets within water, just the opposite. The time $t_{\mathrm{cr}}$ at which this transient regime of $n_{\text {eff }}=2 / 3$ ends is no later than the time when the critical Reynolds number is reached. From Eq. (1) this time can be estimated to be $t_{\mathrm{cr}} \sim \mathrm{Re}_{\mathrm{cr}}^{3}$. The value of $\mathrm{Re}_{\mathrm{cr}}$ is typically of order 10-100 [34], and is dependent on geometry.

While many numerical simulations have reported evidence for $n=2 / 3$ [17-23], it is likely that these simulations have not probed the asymptotic regime. From the capillary mean free path $\eta^{2} / \rho \sigma$ and the size of domains, one can estimate the Reynolds number during the regime where the $2 / 3$ exponent is seen: $\operatorname{Re}=\sqrt{L \rho \sigma / \eta^{2}}$. The maximum Reynolds number reached in simulations [17-22] is no more than [35] 5-10, so $t_{\mathrm{cr}}$ has not been reached. Hence the numerical work does not provide evidence for an asymptotic exponent describing a new universality class, only a transient.

In conclusion, the upper bound on the growth exponent was obtained by assuming an asymptotic scaling regime, in which all lengths scale in the same fashion: Thus, either there is an asymptotic scaling and $n \leq 1 / 2$ or there is no asymptotic scaling [32]. Further work is necessary to determine the nature of asymptotic growth and scaling in the spinodal decomposition of fluids.

This work was supported by the Natural Sciences and Engineering Research Council of Canada (M. G.), le Fonds pour la Formation de Chercheurs et l'Aide à la Recherche du Québec (M. G.) and Research Corporation Grant No. CC4181 (K. R. E.).

[1] J. D. Gunton, M. San Miguel, and P. Sahni, in Phase Transitions and Critical Phenomena, edited by C. Domb and J.L. Lebowitz (Academic Press, London, 1983), Vol. 8, p. 267.

[2] A. J. Bray, Adv. Phys. 32, 357 (1994).

[3] P. C. Hohenberg and B. I. Halperin, Rev. Mod. Phys. 49, 435 (1977).

[4] E. Siggia, Phys. Rev. A 20, 595 (1979).

[5] K. Kawasaki and T. Ohta, Physica (Amsterdam) 118A, 175 (1983).

[6] M. San Miguel, M. Grant, and J. D. Gunton, Phys. Rev. A 31, 1001 (1985).

[7] H. Furukawa, Phys. Rev. A 31, 1103 (1985).

[8] Y.C. Chou and W. I. Goldburg, Phys. Rev. A 23, 858 (1981).

[9] N.-C. Wong and C. M. Knobler, Phys. Rev. A 24, 3205 (1981).

[10] E. D. Siebert and C. M. Knobler, Phys. Rev. Lett. 54, 819 (1985).

[11] P. Guenoun, R. Gastaud, F. Perrot, and D. Beysens, Phys. Rev. A 36, 4876 (1987).
[12] A. Cumming, P. Wiltzius, and F. S. Bates, Phys. Rev. Lett. 65, 863 (1990); A. Cumming, P. Wiltzius, F. S. Bates, and J. H. Rosedale, Phys. Rev. A 45, 885 (1992).

[13] M. Takenaka and T. Hashimoto, J. Chem. Phys. 96, 6177 (1992).

[14] T. Baumberger, F. Perrot, and D. Beysens, Phys. Rev. A 46, 7636 (1992).

[15] F. Perrot, P. Guenoun, T. Baumberger, D. Beysens, Y. Garrabos, and B. Le Neindre, Phys. Rev. Lett. 73, 688 (1994).

[16] V.S. Nikolayev, D. Beysens, and P. Guenoun, Phys. Rev. Lett. 76, 3144 (1996).

[17] J. E. Farrell and O. T. Valls, Phys. Rev. B 40, 7027 (1989).

[18] F. J. Alexander, S. Chen, and D. W. Grunau, Phys. Rev. B 48, 634 (1993).

[19] Y. Wu, F. J. Alexander, T. Lookman, and S. Chen, Phys. Rev. Lett. 74, 3852 (1995).

[20] S. Bastea and J. L. Lebowitz, Phys. Rev. E 52, 3821 (1995).

[21] W. R. Osborn, E. Orlandini, M. R. Swift, J. M. Yeomans, and J. R. Banavar, Phys. Rev. Lett. 75, 4031 (1995).

[22] T. Lookman, Y. Wu, F. J. Alexander, and S. Chen, Phys. Rev. E 53, 5513 (1996). These authors recognize that scale invariance implies that the growth law determines a time-dependent Reynolds number.

[23] H. Furukawa, Phys. Rev. E 55, 1150 (1997). This author notes that $n=1 / 2$ is a special value related to $\operatorname{Re}$ increasing.

[24] P. B. S. Kumar and M. Rao, Phys. Rev. Lett. 77, 1067 (1996).

[25] M. Laradji, S. Toxvaerd, and O. G. Mouritsen, Phys. Rev. Lett. 77, 2253 (1996).

[26] W.-J. Ma, A. Maritan, J. R. Banavar, and J. Koplik, Phys. Rev. A 45, R5347 (1992).

[27] A. Shinozaki and Y. Oono, Phys. Rev. E 48, 2622 (1993).

[28] E. Velasco and S. Toxvaerd, Phys. Rev. Lett. 71, 388 (1993); see also P. Ossadnik, M. F. Gyure, H. E. Stanley, and S. C. Glotzer, Phys. Rev. Lett. 72, 2498 (1994).

[29] G. Leptoukh, B. Strickland, and C. Roland, Phys. Rev. Lett. 74, 3636 (1995).

[30] As to the asymptotic value of $n$, we have no firm conclusions. There are suggestions in the literature [2,20,22,23] for mechanisms giving variously $n=1 / 2$ and $n=1 / 3$. It is also suggested that $n=1 / z$, where $z$ is the slowing down exponent for close-to-equilibrium roughening: See, for example, T. M. Rogers (Ph.D. thesis, University of Toronto, 1988), L. Jörgenson, R. Harris, and M. Grant [Phys. Rev. Lett. 63, 1693 (1989)]for "models A, B, and $C$ " [1,3]. Calculations of the dynamic roughening exponent $z$ for fluid interfacial systems have been done to obtain the dynamic prefactor for nucleation: K. Kawasaki, J. Stat. Phys. 12, 365 (1975); L. A. Turski and J. S. Langer, Phys. Rev. A 22, 2189 (1980). These involve physically motivated approximations, and give $z=3$. However, in a numerical analysis of the eigenvalue spectrum for roughening of fluid interfaces, A. Shinozaki [Phys. Rev. E 48, 1984 (1993)]suggests that the identification of $z$ with $1 / n$ may not be correct in this case. This paper also demonstrates the subtleties involved in extracting $z$ from the eigenvalue spectrum for fluid interfacial systems. 
[31] Effective exponents have been identified by dimensional arguments $[2,20,22,23]$. For early times $\left(L \ll(\eta \sigma)^{1 / 2}\right.$, where $\sigma$ is surface tension), diffusion dominates and $L \sim$ $t^{1 / 3}$. For intermediate times $\left[(\eta \sigma)^{1 / 2} \ll L \ll \eta^{2} / \rho \sigma\right]$, viscous effects dominate giving $L \sim t$ in three dimensions, and $L \sim t^{1 / 2}$ in two dimensions. It is for still later times $\left(L \gg \eta^{2} / \rho \sigma\right)$ that it is argued $L \sim t^{2 / 3}$. Finally, we note that droplet coalescence in off-critical quenches is expected to give $L \sim t^{1 / d}$ in $d$ dimensions.

[32] A. J. Wagner and J.M. Yeomans [Phys. Rev. Lett. 80, 1429 (1998)]note that, in the time regime that they are able to probe numerically, they do not reach a scaleinvariant regime, if one exists. The absence of noise in those simulations has apparently resulted in a very small or zero diffusivity, leading to droplets within droplets which coarsen extremely slowly. Similar results are described by H. Tanaka and T. Araki [Phys. Rev. Lett. 81, 389 (1998)].

[33] In a binary fluid, the concentration $c$ is coupled to $p$ by the Gibbs-Duhem relationship: $\vec{\nabla} p \sim c \vec{\nabla} \mu$, where $\mu$ is the chemical potential. The dynamics of $c$ are coupled to $\vec{v}$ by $\partial c / \partial t+\vec{v} \cdot \vec{\nabla} c=\Gamma \nabla^{2} \mu$, where $\Gamma$ is a mobility. For a curved interface, there are corrections to the chemical potential of the form $\sigma / L$.

[34] L. D. Landau and E. M. Lifshitz, Fluid Mechanics (Pergamon Press, Oxford, 1959).

[35] In Ref. [23] it is reported that $\eta^{2} / \rho \sigma \sim 10^{-4}$ in units of the lattice constant, leading to an estimate of $\operatorname{Re} \sim 10^{3}$. However, it is not possible to simulate length scales smaller than the lattice constant, so that a more realistic estimate of Re involves replacing $\eta^{2} / \rho \sigma$ with one lattice constant, which gives $\operatorname{Re} \sim 10$. 